\title{
Kirschner wire migration from the clavicle to the cervical spine
}

\author{
Jakub Liberski, Krzysztof Ficek
}

\section{ABSTRACT}

Introduction: Kirschner wires (K-wires) are often used to treat injury to the shoulder girdle. Often found in such as literature are cases of migration of $\mathrm{K}$-wires to the thoracic organs lungs, esophagus, aorta. This is a potentially a very serious complication that could lead to death. Case Report: A 31-year-old male patient who was surgically treated for acromioclavicular joint separation on the right side. Six years later, the patient was admitted to the orthopedic department due to severe pain around the right acromioclavicular joint and septic inflammation in the surrounding area. The plain $X$-ray showed that Weber's cerclage was broken and one of the Kirschner wires had migrated towards the central axis of the body, perpendicular to the spine at the level of (the 7 th) cervical vertebra $\left(\mathrm{C}_{7}\right)$. The angio computed tomography test, using multi-helical amplified contrast, showed that the displaced $6.5 \mathrm{~cm}$ long K-wire segment had migrated to the spinal cord canal at the $\mathrm{C}_{7}$ vertebra. The wire passed through the two transverse foramen of $\mathrm{C}_{7}$ and adhered to the posterior surface of the trunk vertebra. The study did not show any pathological symptoms of the nervous system resulting from K-wire migration. The patient did not consent to the removal of the displaced $K$ wire, due to alcoholism. Conclusion: Patients

\section{Jakub Liberski ${ }^{1}$, Krzysztof Ficek ${ }^{2}$}

Affiliations: ${ }^{1} \mathrm{MD}$, Galen-Orthopaedics, Bierun, Poland; ${ }^{2}$ Reviewer, Lecturer, MD, PhD, Galen-Orthopaedics, Bierun, Poland

Corresponding Author: Krzysztof Ficek, Bierun, POLAND. 43-150; Phone: +48 501545 011; Fax No: +48 3221644 02; Email: galen@galen.pl

Received: 24 July 2012

Accepted: 07 December 2012

Published: 01 June 2013 with K-wire osteosynthesis should be under strict control in the outpatient clinic. The $\mathrm{K}$ wires must be bent at the distal end to prevent dislocations and a potentially fatal wire migration. Consider this type of orthopedic fixation in patients not complying with medical recommendations (alcoholism, mental illness, low IQ).

Keywords: Kirschner wires (K-wires), Migration, Shoulder, Acromioclavicular joint, Cervical spine

$* * * * * * * * *$

Liberski J, Ficek K. Kirschner wire migration from the clavicle to the cervical spine. International Journal of Case Reports and Images 2013;4(6):308-311.

$$
* * * * * * * * *
$$

doi:10.5348/ijcri-2013-06-319-CR-4

\section{INTRODUCTION}

The discussion of methods for treating acute acromioclavicular separation is ongoing. Most authors lean towards non-operative treatment on I grade and II grade of Rockwood scale. [1, 2]. In III grade cases, medical opinions begin to diverge as conservative treatment may result in scapular dyskinesis, SICK scapula syndrome, which causes discomfort in the shoulder girdle, weakness, and inability to take part in sporting activities [3]. If there is any doubt as to whether a patient would definitely benefit from surgery, it is reasonable to treat the patient expectantly, and if he has persistent symptoms, then a later stabilization operation may be done [4]. Present operating techniques are mostly based on reconstruction of the coracoclavicular ligaments e.g., TightRope ${ }^{\mathrm{TM}}$, MINAR technique. [5] The other methods used are distal 
clavicle hook plate, Bosworth-type screw and Dacron graft technique. In late unreduced acromioclavicular joint dislocation, reconstructive methods are applied using graft or Weaver and Dunn, Copeland and Kessel methods. One approach for the surgical treatment of an acute acromioclavicular separation is the use of $\mathrm{K}$-wires as a stabilizing element to set the clavicle position in relation to the acromion.

\section{CASE REPORT}

A 31-year-old male patient was admitted to the orthopaedic department due to severe pain around the right acromioclavicular joint and severely limited active and passive range of motion stemming from septic inflammation in the surrounding area. The patient had been surgically treated six years earlier for acromioclavicular joint separation on the right side (III grade of Rockwood classification). Weber's cerclage was applied to maintain reduced dislocation. However, the patient discontinued treatment and refused to have the hardware removed, due to chronic alcoholism.

Acute pain occurred four days prior to admission, immediately after the collapse of the abducted upper extremity. The study case presented a septic fistula in the area of the right acromioclavicular joint and skin tension above the dislocated metallic fusion (Figure 1). No blood supply or innervation disorders of the right upper extremity and right shoulder were observed. Passive and active range of motion of the right upper extremity were limited by pain.

One of the K-wires was broken and its proximally longer segment was displaced above the right acromioclavicular joint. The second segment, with tip intact, had migrated towards the central axis of the body, perpendicular to the spine at the level of the 7 th cervical vertebra $\left(\mathrm{C}_{7}\right)$. The flexible wire between the clavicle and acromion had snapped. The angio computed tomography test, using multi-helical amplified contrast, revealed the second displaced segment of K-wire, with a length of $6.5 \mathrm{~cm}$, in the spinal cord canal at the $\mathrm{C}_{7}$ vertebra. The wire passed through the two transverse foramen of cervical vertebra $\left(\mathrm{C}_{7} / \mathrm{C} 8\right)$ and adhered to the posterior surface of the trunk vertebra, and thus proceeded to the anterior surface of the spinal cord. On the right side, the tip was located 1 cm behind the carotid artery, at the vessel's exit into the transverse foramen (Figure 2 and Figure 3).

Fusion removal was performed, starting from the incision in the old scar by applying lateral half of Roberts approach, which had also been applied in the first operation. After removal of the K-wire, located in the subcutaneous tissue around the acromioclavicular joint, pain subsided, leading to a gradual improvement in range of motion. Surgery was proposed to remove the $\mathrm{K}$-wire from the cervical vertebrae/spine. However, the patient did not consent to the operation. Despite the use of intensive pharmacotherapy, acute alcohol withdrawal symptoms prevailed. The patient left the hospital at his own request, and assumed full responsibility/liability after being informed of the possible consequences of refusing treatment. The patient defaulted from followup after the fusion removal. His clinical condition was assessed again in 2007 when he was admitted to hospital following a beating. No neurological disorders caused by K-wire migration were found at that time. However, his later fate is unknown.

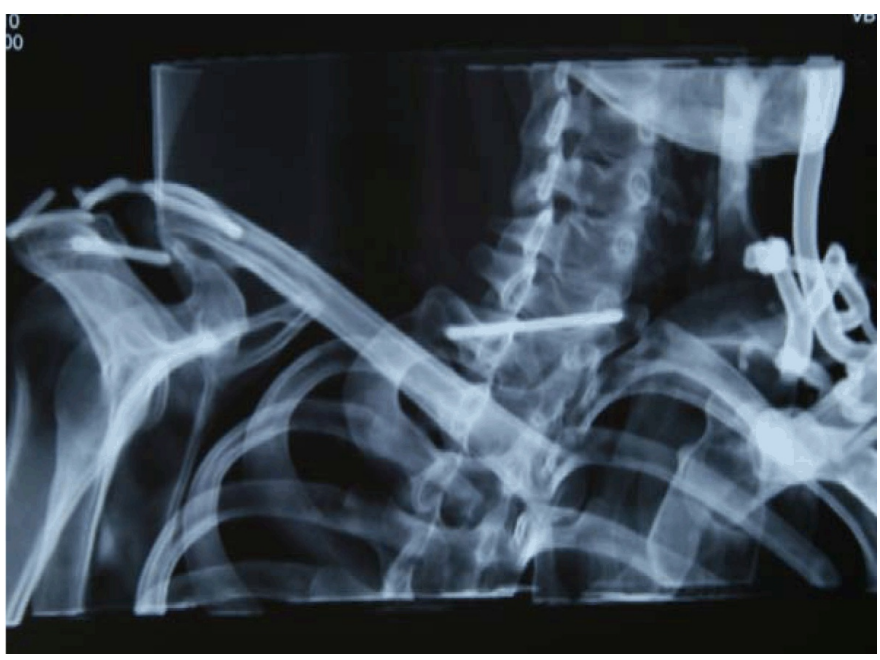

Figure 1: PA X-ray. Migrated K-wire and Weber's cerclage.

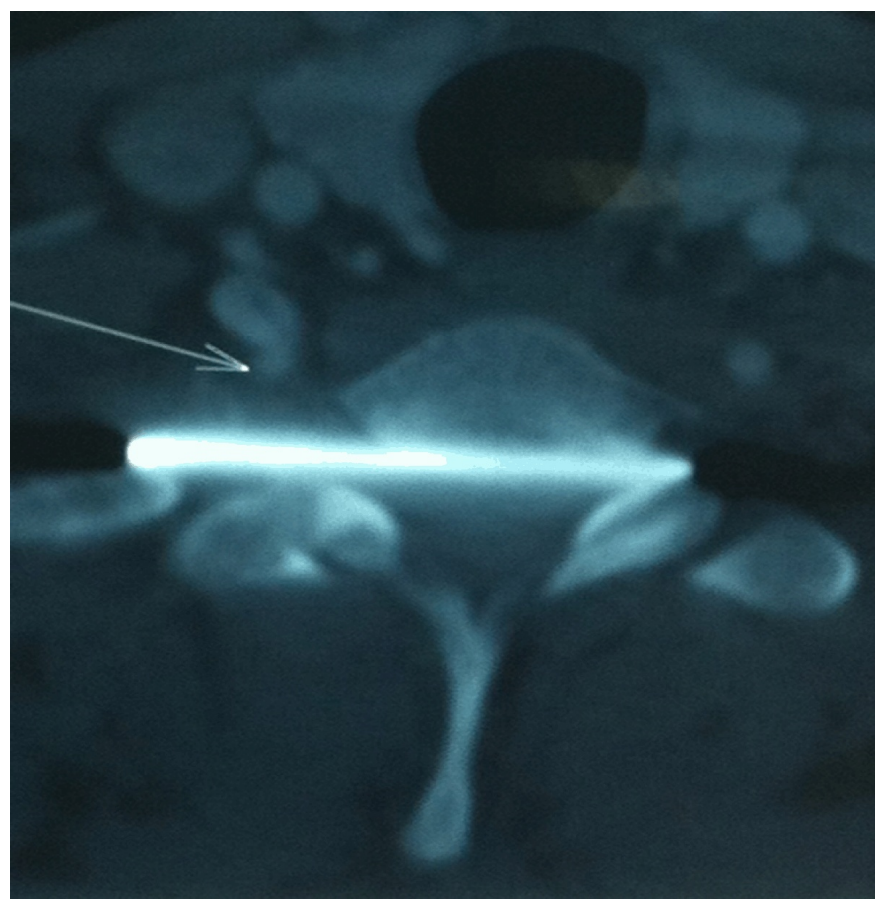

Figure 2: Angio computed tomography scan. The K-wire passed through two transvers foramen of the 7 th cervical vertebra and adhered to the posterior surface of the trunk vertebra, and thus proceeded to the anterior surface of the spinal cord.

\section{DISCUSSION}

In literature, there are numerous cases of migration Kirschner wires used to stabilize fractures and dislocations of the shoulder girdle. The large majority of reports present displacements after clavicular fracture 


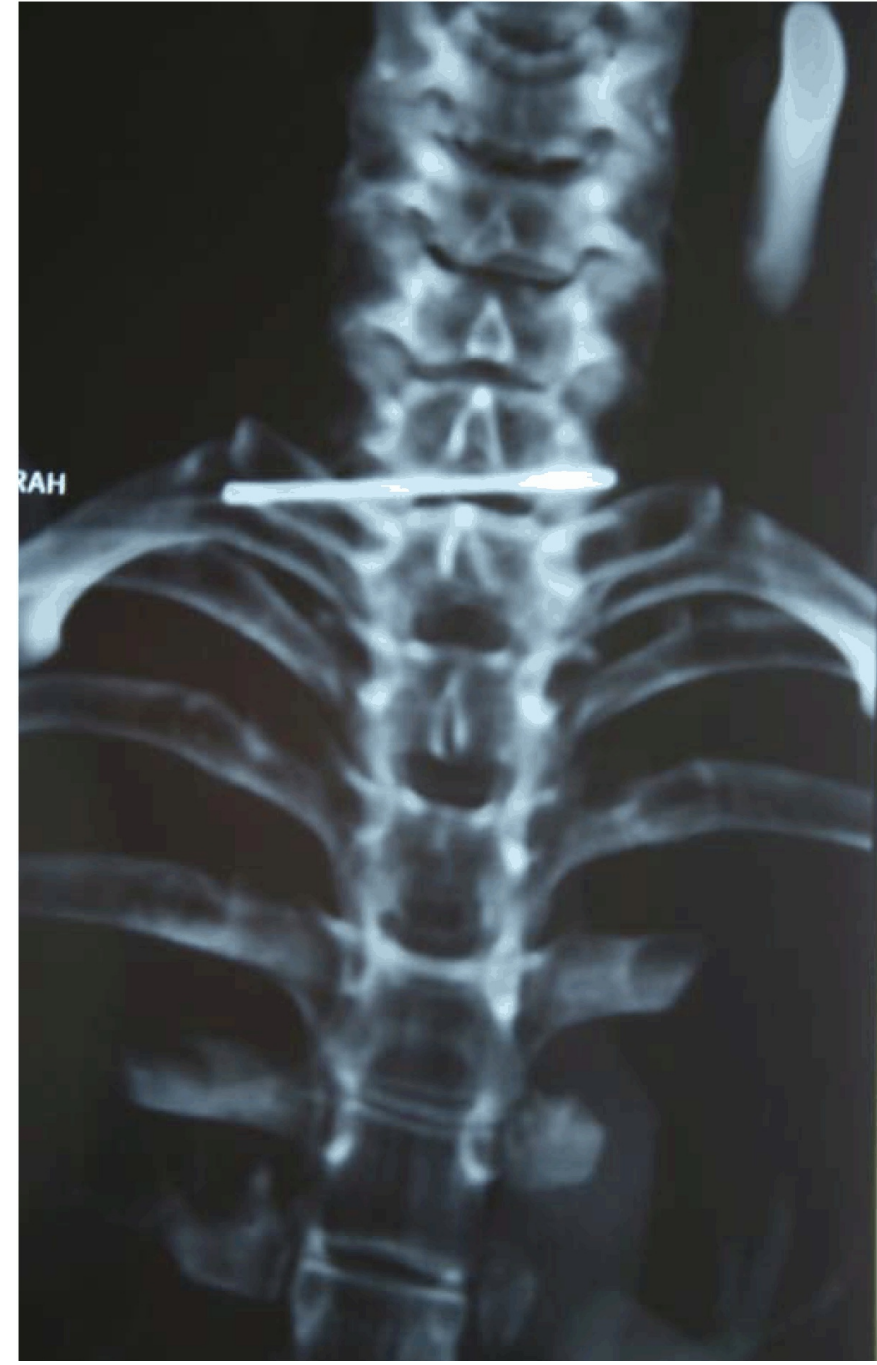

Figure 3: Angio computed tomography scan. AP view shows the level of the migrated wire.

stabilization [6-10], acromioclavicular dislocations [11, 12], and stabilization of fractures closer to the humerus. There have been cases reported of tip migration into the mediastinum, myocardium, lungs, esophagus and spinal cord [6-14].

In this particular case, there was no damage to any important structures, in spite of the tip passing through both $\mathrm{C}_{7}$ intervertebral foramina. However, migrations causing spinal cord damage with Brown-Sequard syndrome are well-documented [10]. To our knowledge, this is the only case of K-wire migration into the front of the spinal cord. It is worth noting that there is an absence of accompanying neurological sequelae. It is impossible to predict whether the migration of the wire has terminated. Its proximity to major blood vessels poses an ongoing threat to the patient's life. In one case, there was migratory damage to the brachio-cephalic trunk, which required immediate surgery [15]. To minimize the risk of migration of the fusion, it is advisable to remove the K-wire after the duration of the time required to heal the injury and to refrain from intensive rehabilitation before removal. By bending the tips, migration can and should be prevented. Most findings concerned complications connected with Kwire migration after fracture, dislocation or separation of the clavicle.

$\mathrm{K}$-wire is a cheap and easily available fusion but it has a propensity to migrate and does not offer rigid fixation. Nowadays, methods based on reconstruction of the coracoclavicular ligaments, e.g, TightRope ${ }^{\mathrm{TM}}$, MINAR technique are preferable [5]. These methods are minimally invasive, provide good stability of acromioclavicular joint and do not call for another fusion removal surgery. However, there is a risk of plexus brachialis damage, pneumothorax, and fracture of the coracoid process. Application of a Bosworth screw may give better result in similar complications.

A distal clavicle hook plates also provides good stabilization, but is more difficult to insert and calls for another operation. Its application may also result in pneumothorax. We opted methods based on reconstruction of the coracoclavicular ligaments due to their low level of invasiveness and high effectiveness. However, the cost of $\mathrm{K}$-wire application is the lowest of the aforementioned methods.

Patients with K-wire osteosynthesis should be under strict control in the outpatient clinic. Regular X-rays should be performed. Patients should be informed about potential complications.

Because of its satisfactory results in the treatment of this type of injury, the historical method of K-wire application is practical and effective. In the event of Kwire application, literature stresses the need to bend the lateral end of the K-wire, a detail which is not always complied with or performed in an appropriate manner.

Consider the risks associated with this type of fixation in patients not complying with medical recommendations (alcoholism, mental illness, low IQ).

\section{CONCLUSION}

Considering the aforementioned case and other cases of K-wire migration from literature, it seems that the opinion of nonsurgical treatment of acromioclavicular separation (Rockwood grades I-III) is reasonable. The right kind of fusion should be individually suited to the demands of the patient.

With less demanding patients (old age, nonathletic, sedentary life-style, non-dominant side, fear of operation, alcohol, drugs, mental subnormaliry) nonsurgical treatment seems to be the right procedure.

$$
* * * * * * * * *
$$

\section{Author Contributions}

Jakub Liberski - Substantial contributions to conception and design, Acquisition of data, Drafting the article, Revising it critically for important intellectual content, Final approval of the version to be published Krzysztof Ficek - Substantial contributions to conception and design, Acquisition of data, Drafting the article, Revising it critically for important intellectual content, Final approval of the version to be published 


\section{Guarantor}

The corresponding author is the guarantor of submission.

\section{Conflict of Interest}

Authors declare no conflict of interest.

\section{Copyright}

(C) Jakub Liberski et al. 2013; This article is distributed under the terms of Creative Commons Attribution 3.0 License which permits unrestricted use, distribution and reproduction in any means provided the original authors and original publisher are properly credited. (Please see www.ijcasereportsandimages.com /copyright-policy.php for more information.)

\section{REFERENCES}

1. Reid D, Polson K, Johnson L. Acromioclavicular joint separations grades I-III: A review of the literature and development of best practice guidelines. Sports Med 2012 Aug 1;42(8):681-96.

2. Smith TO, Chester R, Pearse EO, Hing CB. Operative versus non-operative management following Rockwood grade III acromioclavicular separation: A meta-analysis of the current evidence base. J Orthop Traumatol 2011 Mar;12(1):19-27.

3. Murena L, Canton G, Vulcano E, Cherubino P. Scapular dyskinesis and SICK scapula syndrome following surgical treatment of type III acute acromioclavicular dislocations. Knee Surg Sports Traumatol Arthrosc 2013 May;21(5):1146-50.

4. Allman FL Jr. Fractures and ligamentous injuries of the clavicle and its articulation. J Bone Joint Surg Am 1967 Jun;49(4):774-84.

5. Wellmann M, Smith T. Epidemiology, anatomy, biomechanics and imaging of acromioclavicular joint injuries. Unfallchirurg 2012 Oct;115(10):867-1. [[Article in German].

6. Fransen P, Bourgeois S, Rommens J. Kirschner wire migration causing spinal cord injury one year after internal fixation of a clavicle fracture. Acta Orthopaedica Belgica 2007 Jun;73(3):390-2.

7. Nishizaki K, Seki T. Intracardiac migration of a Kirschner wire from the right clavicle. Asian Cardiovasc Thorac Ann 2007 Jun;15(3):272-3.

8. Pribán V, Toufar P. A spinal cord injury caused by a migrating Kirschner wire following osteosynthesis of the clavicle: A case review. Rozhl Chir 2005 Jul;84(7):373-5. [Article in Czech].

9. Hegemann S, Kleining R, Schindler HG, Holthusen $\mathrm{H}$. Kirschner wire migration in the contralateral lung after osteosynthesis of a clavicular fracture. Unfallchirurg 2005 Nov;108(11):991-3. [Article in German].

10. Regel JP, Pospiech J, Aalders TA, Ruchholtz S. Intraspinal migration of a Kirschner wire 3 months after clavicular fracture fixation. Neurosurgical Review 2002 Mar;25(1-2):110-2.

11. Gulcan O, Sezgin AT, Bolat B, Turkoz R. Right ventricular penetration and cardiac tamponade as a late complication of Kirschner wire placement in the sternoclavicular joint. Interact Cardiovasc Thorac Surgery 2005 Aug;4(4):295-6.

12. Durpekt R, Vojácek J, Lischke R, Burkert J, Spatenka J. Kirschner wire migration from the right sternoclavicular joint to the heart: A case report. Heart Surgery Forum 2006;9(6):E840-2.

13. Freund E, Nachman R, Gips H, Hiss J. Migration of a Kirschner wire used in the fixation of a subcapital humeral fracture, causing cardiac tamponade: Case report and review of literature. The American Journal Forensic Medicine and Pathology 2007 Jun;28(2):155-6.

14. Loncán LI, Sempere DF, Ajuria JE. Brown-Sequard syndrome caused by a Kirschner wire as a complication of clavicular osteosynthesis. Spinal Cord 1998 Nov;36(11):797-9.

15. Wada S, Noguchi T, Hashimoto T, Uchida Y, Kawahara K. Successful treatment of a patient with penetrating injury of the esophagus and brachiocephalic artery due to migration of Kirschner wires. Annals of Thoracic Cardiovascular Surgery 2005 Oct;11(5):313-5.
Access full text article on other devices

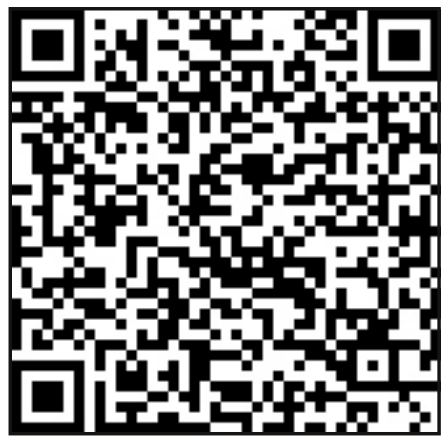

Access PDF of article on other devices

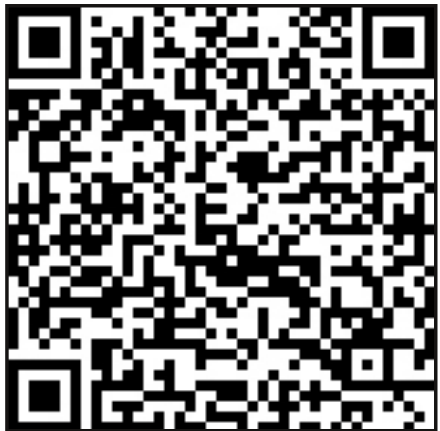

